\title{
A study on the influence of prosthetic interface material in transtibial amputees' gait
}

\author{
Adriana Cavaco $^{\mathrm{a}, \mathrm{b}, *}$, Luísa Durães ${ }^{\mathrm{b}}$, Amílcar Ramalho $^{\mathrm{c}}$ and Sandra Pais ${ }^{\mathrm{d}}$ \\ ${ }^{a}$ ESSUAlg, School of Health, University of Algarve, Portugal \\ ${ }^{\mathrm{b}}$ CIEPQPF, Department of Chemical Engineering, University of Coimbra, Portugal \\ ${ }^{\mathrm{C}}$ CEMMPRE, Department of Mechanical Engineering, University of Coimbra, Portugal \\ ${ }^{\mathrm{d}}$ CBMR, Department of Biomedical Sciences and Medicine, University of Algarve, Portugal
}

Received 12 July 2018

Accepted 26 June 2020

\begin{abstract}
.
BACKGROUND: Interfaces of transtibial prosthesis have an important role in the transmission of ground reaction forces, damping gait loads and tissue protection.

OBJECTIVE: This work aims to study the influence of prosthesis interfaces on amputees' gait and perception on pain, comfort and the overall interface/prosthesis system.

METHODS: Commercial samples of three different interface materials were selected: block copolymer (Material I), silicone gel (Material II) and silicone elastomer (Material III). Using standardized prosthesis, four amputee subjects performed gait tests at three imposed cadences in a barometric platform. The subjects also filled in a questionnaire about their perception.

RESULTS: Material II presented the highest asymmetries of vertical ground reaction forces and was perceived as the most painful and uncomfortable. Material III led to the most symmetric load distribution between legs and was perceived as the most comfortable. Material I showed better overall biomechanical behavior and better subject's perception.

CONCLUSIONS: Material III showed appropriate characteristics for lower levels of activity for which it is recommended. Prescription of Material II should be rethought, as it is only adequate for specific and occasional situations. Material I should be considered adequate for both active and less active amputees. When correlating the subjects' perception with the interface properties, we concluded that the mechanical properties are the most influential.
\end{abstract}

Keywords: Limb prosthesis, gait analysis, transtibial amputees, materials testing, silicone, block copolymer

\section{Introduction}

The main purpose of the lower limb amputee's rehabilitation is to recover the subject's ability to perform activities of daily living, quality of life and normal gait [1]. Transtibial amputees lose static support and adequate gait function, requiring joints adaptation and muscular mechanisms of compensation [2,3].

\footnotetext{
* Corresponding author: Adriana Cavaco (PhD), School of Health, University of Algarve, Campus de Gambelas Ed 1, 8005139 Faro, Portugal. Tel.: +351 289800 100; E-mail: aicavaco@ualg.pt.
} 
Symmetric limb weight is very important for balance during gait, since the gravity centre is shifted laterally to the sound limb side. Gait asymmetry is imposed by insufficient prosthesis mass to compensate the lost limb weight, loss of normal neuromuscular control, mechanical asymmetries of the prosthesis $[3,4]$ and other factors, like pain and postural instability $[5,6]$.

The quality of prosthetization depends on the choice of components of the prosthesis according to each patient's characteristics. Currently, there is a wide range of available components and a suitable choice therefore requires a deep knowledge of the materials in terms of their structure, properties and behavior [7].

The amputated limb becomes vulnerable due to the absence of joints and tissues for cushioning the impact of the repetitive load during gait, which can induce stump pain [8], discomfort and skin injuries [9], but also knee osteoarthritis in the sound leg, osteoporosis in the stump [10] and back pain [8]. To improve the amputee's safety and comfort, a cushioning interface, usually named a liner, between the stump skin and the prosthetic rigid socket is used, and is a determinant component of the prosthesis [9].

Silicone-based materials and polymeric elastomers have been used in the interface confection and their properties have been studied. The results in the literature show that physical properties of silicone gel and silicone elastomers are quite similar [11,12]. Moreover, elastomers are often preferred due to their stiffness properties and compressive strength, while polyurethane shows higher coefficient of friction [11,12]. The results of the shear tests led to the conclusion that silicone elastomers and polyurethane are the most suitable materials for stumps with a high proportion of soft tissue, because they prevent the slide of the stump in the socket, while silicone gels are appropriate for stumps with abundant bony prominences, due to the cushioning effect $[11,12]$.

Thus, the assessment of rigidity and damping characteristics of the interface material is a determinant step to optimize the interface material selection and adapt its geometry to the needs of the amputee.

Currently, an amputee's functionality is defined based on a Mobility Scale (Functional Levels defined by the Centres for Medicare and Medicaid Services of the United States Department of Health and Human Services [13,14]), which is composed of five K-levels of mobility, from K0 - the patient is immobile and has neither the potential nor the ability to ambulate or transfer, with or without assistance; to K4 - the patient has the ability or potential to use a prosthesis as an aid in activities that require skills beyond basic and are characterised by high impact, energy consumption or stress levels [13,14]. Through this Mobility Scale, Mobility Scale-based Guidelines for prosthetic device provision were developed by the same centres [13,14]. This scale also has five K-levels with information regarding the adequate prosthetic device provision, namely: K0 - patients are not advised to use prosthesis either for ambulation or transfer; $\mathrm{K} 1$ - patients are advised to use prosthesis to attain the functional goal of unlimited or limited in-house ambulation; K2 - patients are advised to use prosthesis so as to attain the functional goal of limited community ambulation; K3 - patients are advised to use prosthesis in order to develop their functional potential that goes beyond mere locomotion, so as to be able to traverse most barriers and cope with more demanding exercises and other activities; K4 - patients (typically children, active adults and athletes) are advised to use prosthesis even with the most demanding activities [13,14]. Nowadays, this classification method is used by manufacturers to classify the suitability of prosthesis components according to the patient's functional level [13].

The aim of this research was to study the influence of the prosthetic interface material in transtibial amputees' gait and perception on pain, comfort and the interface/prosthesis system. For this purpose, three commercial samples of interfaces, representative of the most used elastomeric materials, were studied in their service conditions (in vivo). The biomechanical behavior and subject perception results were analysed considering also the materials mechanical and structural characteristics, previously evaluated 


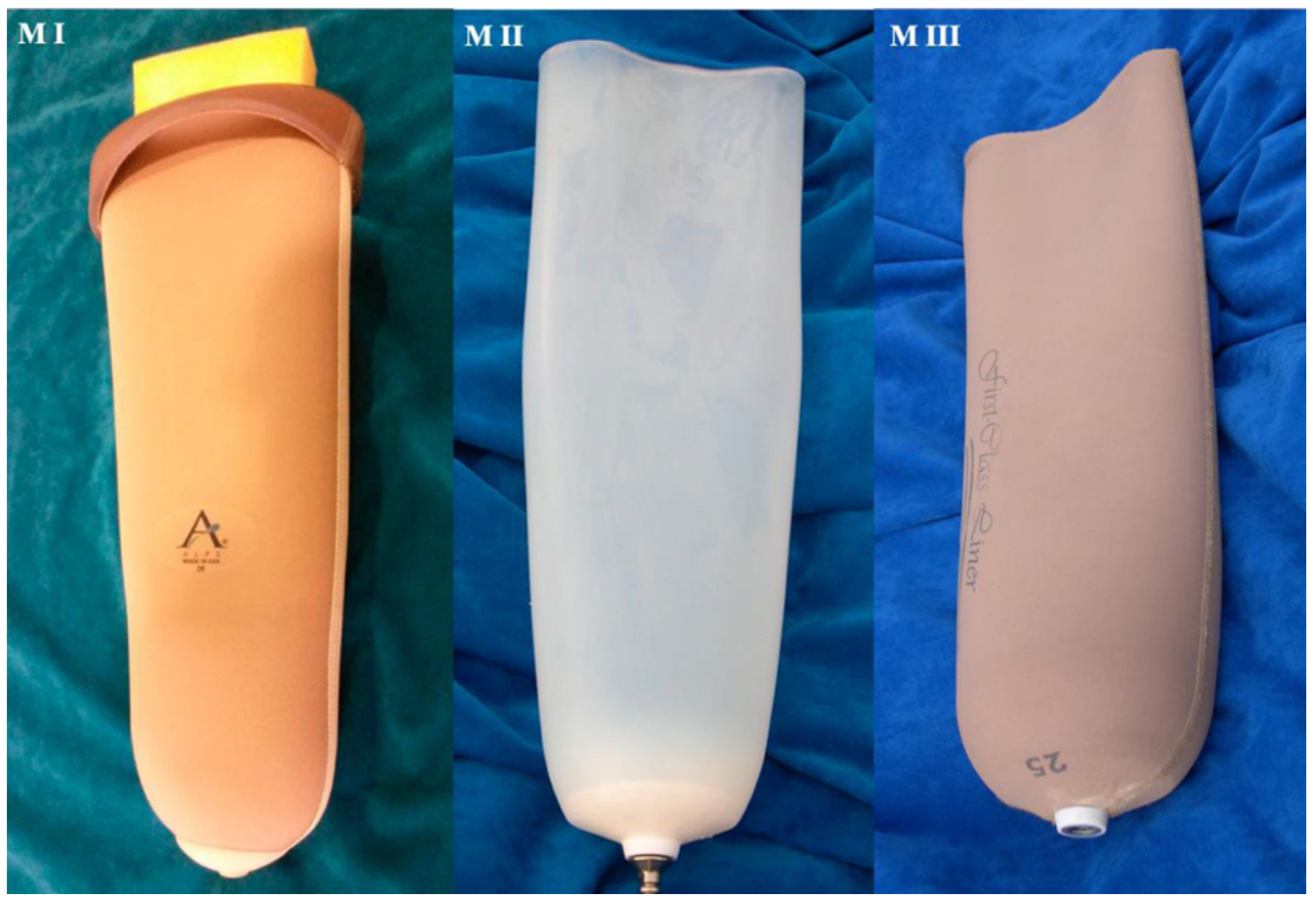

Fig. 1. Studied materials: M I) a block copolymer (AKDT Locking, Extreme gel for high activity); M II) a silicone gel (Clear liner basic, Streifeneder 1S3 silicone gel), and M III) a silicone elastomer (First class liner, Streifeneder 1S5 silicone elastomer).

by the authors $[15,16]$. We believe that the knowledge of the mentioned influence may draw attention to the essential importance of this component and their properties and result in an adequate precursor of prescribing guidelines, which are currently non-existent.

\section{Methods}

\subsection{Interface materials}

Three of the most used commercial interface materials were studied (Fig. 1): Material I, a block copolymer (AKDT Locking, Extreme gel for high activity); Material II, a silicone gel (Clear liner basic, Streifeneder 1S3 silicone gel) and Material III, a silicone elastomer (First class liner, Streifeneder 1S5 silicone elastomer).

According to the Mobility Scale-based Guidelines for prosthetic device provision described in Section 1, interfaces of Materials I and II are recommended for more active amputees (K3-K4), while Material III is recommended for less active amputees (K1-K2).

\subsection{Study design and preparation}

In order to characterize the materials in their service conditions (in vivo), it was necessary to assess their biomechanical behavior in transtibial amputees during a gait course, where kinematic and kinetic 
Table 1

Characterization of the subjects with amputation who voluntarily participated in the test study of the interfaces

\begin{tabular}{lcccc}
\hline & \multicolumn{4}{c}{ Subject } \\
\cline { 2 - 5 } & A & B & C & D \\
\hline Age (years) & 34 & 42 & 52 & 54 \\
Weight (kg) & 80 & 82 & 85 & 85 \\
Height (cm) & 183 & 177 & 180 & 183 \\
Time of amputation (years) & 10 & 14 & 35 & 18 \\
Amputation side & Right & Right & Left & Right \\
Activity level & High & Moderate & Moderate & High \\
Stump shape & Conical & Conical & Conical & Cylindrical \\
Soft tissue & Firm & Firm & Regular & Flaccid \\
Bone prominences & Fibula head & None & Fibula head & None \\
Skin condition & Preserved & Preserved & Preserved & Preserved \\
Blood circulation & Preserved & Preserved & Preserved & Preserved \\
Tolerance to distal pressure & Moderate & High & High & High \\
\hline
\end{tabular}

data were collected. The information of the individual's perception about the prosthesis, pain and comfort for each interface was also collected through a questionnaire.

This study involved several phases. First, an approval of a local ethics committee was obtained, and after that, subjects with transtibial amputation were selected. All the selected subjects visited the prosthesis laboratory three times: first for making the socket casts, preparing the prosthesis and filling in the characterization questionnaire; second for testing the prosthetic sockets and alignments and recording the self-selected walking cadences (slow, normal and fast); and third for assessing the gait and filling in the Global Perception Questionnaire.

\subsubsection{Subject selection}

The subjects were selected from the list of patients of the Instituto de Especialidades Ortopédicas S.L. (IDEO) and were asked to participate voluntarily in this study, based on the following inclusion criteria: i) unilateral traumatic transtibial amputation ( $>2$ years); ii) males under the age of 55; iii) stump length between middle and lower third; iv) stable stump with skin integrity preserved; v) no associated pathologies.

Four male patients ( $45.5 \pm 9.3$ years old) agreed to participate in the study. They all had a high/moderate activity level and the stumps were in good conditions in terms of shape, skin, blood circulation and distal pressure tolerance. For each patient, a characterization form was filled in with personal and amputation data (Table 1). All subjects were willing to participate freely in this study and signed an informed consent form.

\subsubsection{Prosthesis preparation and characteristics}

In order to limit bias and eliminate the influence of other prosthesis components on gait, which has been previously cited as a limitation in these kind of studies [4], an endoskeletal prosthesis was prepared with the same modular prosthesis components (Fig. 2a). Moreover, the same uniformed running shoes 


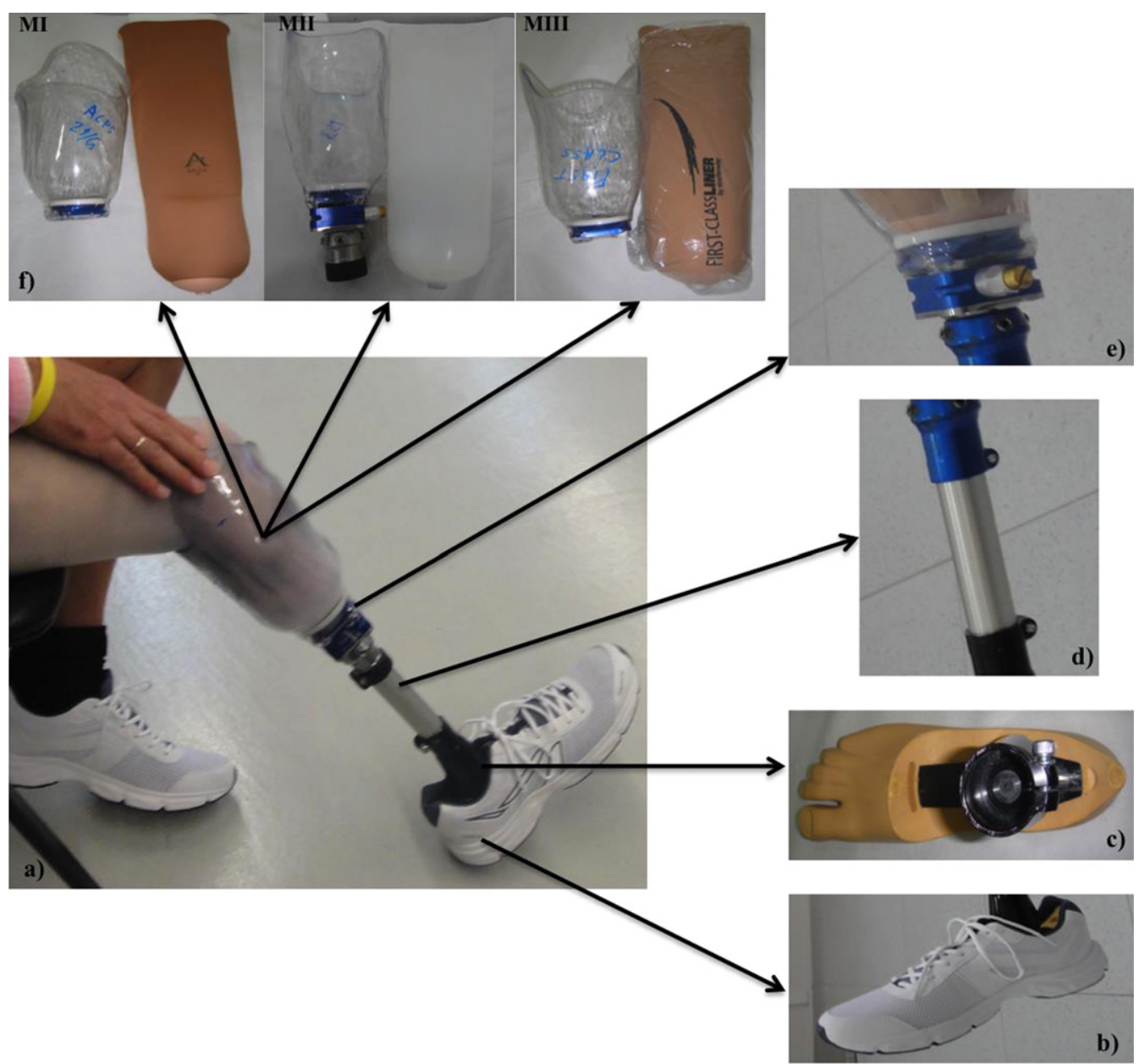

Fig. 2. Prosthesis components description: (a) complete endoskeletal prosthesis; (b) running shoe; (c) prosthetic foot; (d) rigid pylon; (e) pin lock system; (f) interfaces used in the study (Materials I, II and III) and matching full contact sockets.

were used for all subjects (Fig. 2b). The selected prosthetic foot was a Multiflex-standard ${ }^{\circledR}$ by Endolite (Fig. 2c) with a duralumin rigid pylon (Fig. 2d) and pin lock system (Fig. 2e). Also, three different full contact sockets for each of the three interfaces were made per subject (twelve in total), in a transparent thermoplastic material (Fig. 2f). This procedure is fundamental, since the rigid components must be as adapted as possible to the stump of the ampute, in order to provide the structure and stability necessary for the remaining prosthesis. In this sense, the fittings of the full contact sockets must be manufactured in an individualized way, considering the anatomical characteristics of each subject, as well as the interface being applied. For standardized manufacturing, the cast was made with the aid of a pressure machine, so that the forces applied in the removal of the cast were the same in the three interfaces.

At the first lab visit, the conditions of the stump were evaluated and measures were taken in accordance with standard procedures and a datasheet used by IDEO, and the casts were made and the size of some prosthetic components were selected, as the foot/shoe size and the rigid pylon height. 


\begin{tabular}{|l|c|l|}
\multicolumn{3}{|c|}{ Subject's walkway stetup } \\
\hline & Barometric platform FreeMed $\mathbb{}$ & \\
\hline & & \\
\hline & 1.2 meters & \\
\hline & & \\
\hline
\end{tabular}

Fig. 3. Scheme of the experimental setup of the gait test.

Table 2

Order of testing of the interface materials (Material I: a block copolymer; Material II: a silicone gel; and Material III: a silicone elastomer) during the three moments of the gait assessment

\begin{tabular}{lccc}
\hline Subject & $\begin{array}{c}\text { Test moment } 1 \\
\text { First interface tested }\end{array}$ & $\begin{array}{c}\text { Test moment 2 } \\
\text { Second interface tested }\end{array}$ & $\begin{array}{c}\text { Test moment 3 } \\
\text { Third interface tested }\end{array}$ \\
\hline A & Material I & Material II & Material III \\
B & Material II & Material I & Material III \\
C & Material II & Material III & Material I \\
D & Material III & Material II & Material I \\
\hline
\end{tabular}

At the second lab visit, the prosthesis and alignments were tested and the self-selected walking cadences were recorded. Because speed is known to influence the ground reaction force (GRF) [17], the walking cadence was normalized for all subjects. The self-selected slow, normal and fast walking cadences were collected for all subjects after five trails and averaged between all subjects.

All casts and sockets were performed by an experienced certified prosthetist orthotist $(>20$ years of practice), who also performed all prosthesis alignments before each evaluation, to ensure the same alignment, since this has also been found to influence gait [18].

\subsection{Gait assessment}

Gait analysis was carried out at the third lab visit of the subjects, at three imposed cadences over a 10 meters distance, where a barometric platform (FreeMed ${ }^{\circledR}$ Professional Model by Sensor Medica, $200 \mathrm{~Hz}$, $120 \times 50 \mathrm{~cm}^{2}$ of sensors area) was embedded in the middle of the walkway in order to avoid effects of initial acceleration and final deceleration [19] (Fig. 3). A metronome was used to impose, to all subjects, the same average slow ( 75 steps per minute), normal ( 85 steps per minute) and fast (110 steps per minute) cadences during the gait tests. The same sequence was used in all tests: slow, normal and fast. The gait analysis was performed at the Instituto de Biomecánica y Postura de Sevilla.

Five valid trails were collected for each cadence and interface. The sequence order in which the interfaces were tested was randomized (Table 2). Between interfaces, a rest period of fifteen minutes was allowed, during which pain and comfort levels were registered and a Global Perception Questionnaire (GPQ) of the interface was filled in.

Gait tests were preceded by an initial rest period of 30 minutes without any prosthesis, after which warm-up exercises were performed, consisting of repetitions of flexion and extension of the stump and stretching. The gait output parameters were: (1) Step length $(\mathrm{cm})$, measured by the difference in length 
between the prosthetic leg and the sound leg; (2) Stance, swing and double support time (ms), being the average of all cadences and all subjects determined in both legs, according to the interface material used; (3) Vertical component of ground reaction force (GRF-V), being the maximum force of this component collected and normalized with the body weight percentage (\% BW).

\subsection{Pain/comfort perception and Global Perception Questionnaire (GPQ) about the interface/prosthesis system}

All subjects were asked their opinion regarding pain and comfort sensation, compared to their daily prosthesis both at the beginning and ending of all tests, with a visual analogue pain scale $(0=$ no pain; 1 $=$ slightly painful; $2=$ moderately painful and $3=$ very painful $)$ and visual analogue comfort scale $(0=$ very comfortable; 1 = comfortable, $2=$ uncomfortable; 3 = very uncomfortable).

A GPQ was developed to register the subject's perception of the interface/prosthesis. For each tested interface, eight different questions were asked and answered according to a four- point scale:

1. Stand up comfort (very comfortable, comfortable, uncomfortable or very uncomfortable);

2. Sit down comfort (very comfortable, comfortable, uncomfortable or very uncomfortable);

3. Energy required to use the prosthesis (not exhausting, slightly exhausting, exhausting or very exhausting);

4. Instability during walking (never, sometimes, many times or always);

5. Sensation/sensitivity regarding temperature and texture (pleasant, slightly unpleasant, unpleasant or very unpleasant);

6. Easy donning and doffing the interface (easy, slightly difficult, difficult or very difficult);

7. Sweat rate compared with own prosthesis (no sweat, slightly sweaty, sweaty or very sweaty);

8. Prosthesis weight (very light, light, heavy or very heavy).

\subsection{Data analysis}

For each parameter, the applied statistics are described below.

1. Step length: the average of all subjects for each of the three materials was calculated separately for the two legs. The results were split into three groups - Material I, Material II and Material III - and a Mann-Whitney $U$ test was performed regarding the prosthetic and sound leg results.

2. Temporal parameters (swing, stance and double support time): the average of all subjects for each of the three materials was calculated separately for the two legs. The same Mann-Whitney U test was performed as described in item 1 (step length). In addition, a Kruskal-Wallis test was performed, using the results split in three groups (Material I, Material II and Material III), regarding the three gait temporal parameters. Furthermore, a post hoc test (the Bonferroni test) was performed for the values which showed statistical significance.

3. GRF-V: the average of all subjects for each of the three materials was calculated separately for the two legs, with slow, normal and fast cadences. The same Mann-Whitney $U$ test was performed as described before. Additionally, a Kruskal-Wallis test was also performed, with the results split in three groups (Material I, Material II and Material III), regarding the three gait cadences (slow, normal and fast). After that, a post hoc test (the Bonferroni test) was performed for the values which showed statistical significance.

4. The subjects' perception of pain and comfort and their answers to the Global Perception Questionnaire were analyzed and the averages of all subjects for each of the three materials were calculated. The 
Table 3

Step lengths $(\mathrm{cm})$ of the prosthetic and sound legs and difference between them for the three interface materials. Average \pm standard deviation

\begin{tabular}{lccc}
\hline & Prosthetic leg & Sound leg & $\begin{array}{c}\text { Difference } \\
\text { between legs }\end{array}$ \\
\hline Material I & $57.5 \pm 10.2$ & $54.3 \pm 9.3$ & 3.3 \\
Material II & $59.8 \pm 9.4$ & $55.4 \pm 9.7$ & 4.4 \\
Material III & $52.1 \pm 5.7$ & $55.8 \pm 9.7$ & -3.7 \\
\hline
\end{tabular}

differences were assessed for the three interface materials, which were tested in four subjects, using the Kruskal-Wallis test and Bonferroni test. Differences were considered statistically significant when $\mathrm{p}<$ 0.05 .

\section{Results}

All subjects showed slight differences between step length of the prosthetic leg and the sound leg; when using Materials I and II, the results indicate shorter steps in the sound leg, while for Material III the contrary was observed, as shown in Table 3. However, the statistical tests show that there is no significant difference between the legs in the three materials.

Average times (ms) of swing and stance phases in the prosthetic and sound legs and average double support time (ms) over all cadences were calculated (Table 4). Regardless of the material, the stance phase time of the sound leg was always higher than the stance phase in the prosthetic leg. In the prosthetic leg, Material III led to a longer stance phase and shorter double support time. Contrarily,, Material II had a higher swing time of the prosthetic leg and longer double support time. The applied statistical tests showed that there is no significant difference between the legs with the three materials. On the other hand, the Kruskal-Wallis test performed between the three gait temporal phases presented $p$-values $<0.05$ for all materials, showing the statistical significance in these differences. For these results, the Bonferroni test showed $p$-values $<0.05$ between all phases: swing and double support, stance and double support; and swing and stance time, in all materials.

The GRF-V for the three tested materials at slow, normal and fast cadences for prosthetic and sound legs is presented in Table 5. The GRF-V increases slightly as the cadence increases, in all tested materials and in both legs. GRF-V was higher in the sound leg in comparison with the prosthetic leg, for all walking cadences and materials. In general, Material I led to the highest GRF-V at all walking cadences for both legs. On the other hand, Material III showed a more global symmetry between legs, especially at fast cadence.

As before, the statistical tests showed that there is no significance of the differences between the legs with the three materials, but the Kruskal-Wallis performed between the three gait cadences presented a $p$-value $<0.05$ for Material II, showing statistical significance of the recorded difference. For these results, the Bonferroni test showed a statistical significance of the differences between the slow and normal cadences $(p$-value $=0.000)$, slow and fast cadences $(p$-value $=0.018)$ and normal and fast cadences $(p$ value $=0.000)$. 
Table 4

Swing and stance phase times (ms), double support time (ms) and corresponding gait cycle percentage $(\%)$ for the three interface materials. Average \pm standard deviation

\begin{tabular}{lcccccc}
\hline & \multicolumn{2}{c}{ Swing phase time } & & \multicolumn{2}{c}{ Stance phase time } & $\begin{array}{c}\text { Double } \\
\text { support time }\end{array}$ \\
\cline { 2 - 3 } & Prosthetic leg & Sound leg & & Prosthetic leg & Sound leg & \\
\hline Material I & $650.0 \pm 40.6$ & $358.0 \pm 88.6$ & & $685.6 \pm 155.2$ & $738.7 \pm 166.3$ & $198.7 \pm 98.8$ \\
& $48.7 \%$ & $32.6 \%$ & & $51.3 \%$ & $67.4 \%$ & $8.2 \%$ \\
Material II & $648.3 \pm 70.1$ & $481.3 \pm 19.8$ & & $670.2 \pm 127.3$ & $730.3 \pm 161.4$ & $267.7 \pm 85.2$ \\
& $49.2 \%$ & $39.7 \%$ & & $50.9 \%$ & $60.3 \%$ & $10.6 \%$ \\
Material III & $659.5 \pm 38.9$ & $405.8 \pm 83.7$ & & $700.4 \pm 157.5$ & $736.1 \pm 167.9$ & $161.6 \pm 99.2$ \\
& $48.5 \%$ & $35.5 \%$ & $51.5 \%$ & $64.5 \%$ & $6.5 \%$ \\
\hline
\end{tabular}

Table 5

GRF-V ( $\%$ BW) for the different walking cadences and the three interface materials. Average \pm standard deviation

\begin{tabular}{lcccccccc}
\hline & \multicolumn{2}{c}{ Slow cadence } & & \multicolumn{2}{c}{ Normal cadence } & & \multicolumn{2}{c}{ Fast cadence } \\
\cline { 2 - 3 } & $\begin{array}{c}\text { Prosthetic } \\
\text { leg }\end{array}$ & Sound leg & & $\begin{array}{c}\text { Prosthetic } \\
\text { leg }\end{array}$ & Sound leg & & $\begin{array}{c}\text { Prosthetic } \\
\text { leg }\end{array}$ & Sound leg \\
Material I & $129.2 \pm 9.4$ & $131.6 \pm 9.6$ & & $133.8 \pm 8.4$ & $136.0 \pm 8.2$ & & $134.4 \pm 7.8$ & $137.5 \pm 7.2$ \\
Material II & $125.0 \pm 5.7$ & $127.0 \pm 7.1$ & & $130.7 \pm 10.8$ & $134.1 \pm 7.8$ & & $133.6 \pm 14.2$ & $137.9 \pm 8.5$ \\
Material III & $123.1 \pm 7.2$ & $125.1 \pm 10.3$ & & $132.4 \pm 13.5$ & $133.0 \pm 5.7$ & & $134.5 \pm 4.9$ & $134.4 \pm 12.4$ \\
\hline
\end{tabular}

Both pain and comfort were worse after gait testing, regardless of the interface material (Table 6). Material II was identified by all subjects as more painful and less comfortable after gait tests. Material I was perceived as being the one that provides less global pain increase, however, Material III was identified with a more homogeneous behavior providing the smaller global comfort decrease.

Table 6 summarizes the results of the subjects' global opinion on the interface material/prosthesis. Material II was identified as being the worst by all subjects. Materials I and III had similar results in stand up and sit down comfort, sweat rate and prosthesis weight. However, Material I was perceived as being less exhausting regarding the energy required to use the prosthesis, more stable and with easy donning and doffing; and Material III was perceived as the more pleasant on temperature and texture relatively to skin contact.

\section{Discussion}

The difference between the step length of the sound limb and prosthetic limb is common in lower limb amputee's gait. Knee and hip osteoarthritis are common in the transtibial amputee's leg. Step asymmetry is known to cause several problems in the subject's health, like pain, postural instability or osteoarthritis in the contralateral limb. The inadequate prosthesis prescription induces the observed asymmetry. 
Table 6

Pain and comfort, and subjects' global opinion on the interface material/prosthesis. Scale levels are described in Section 2.4

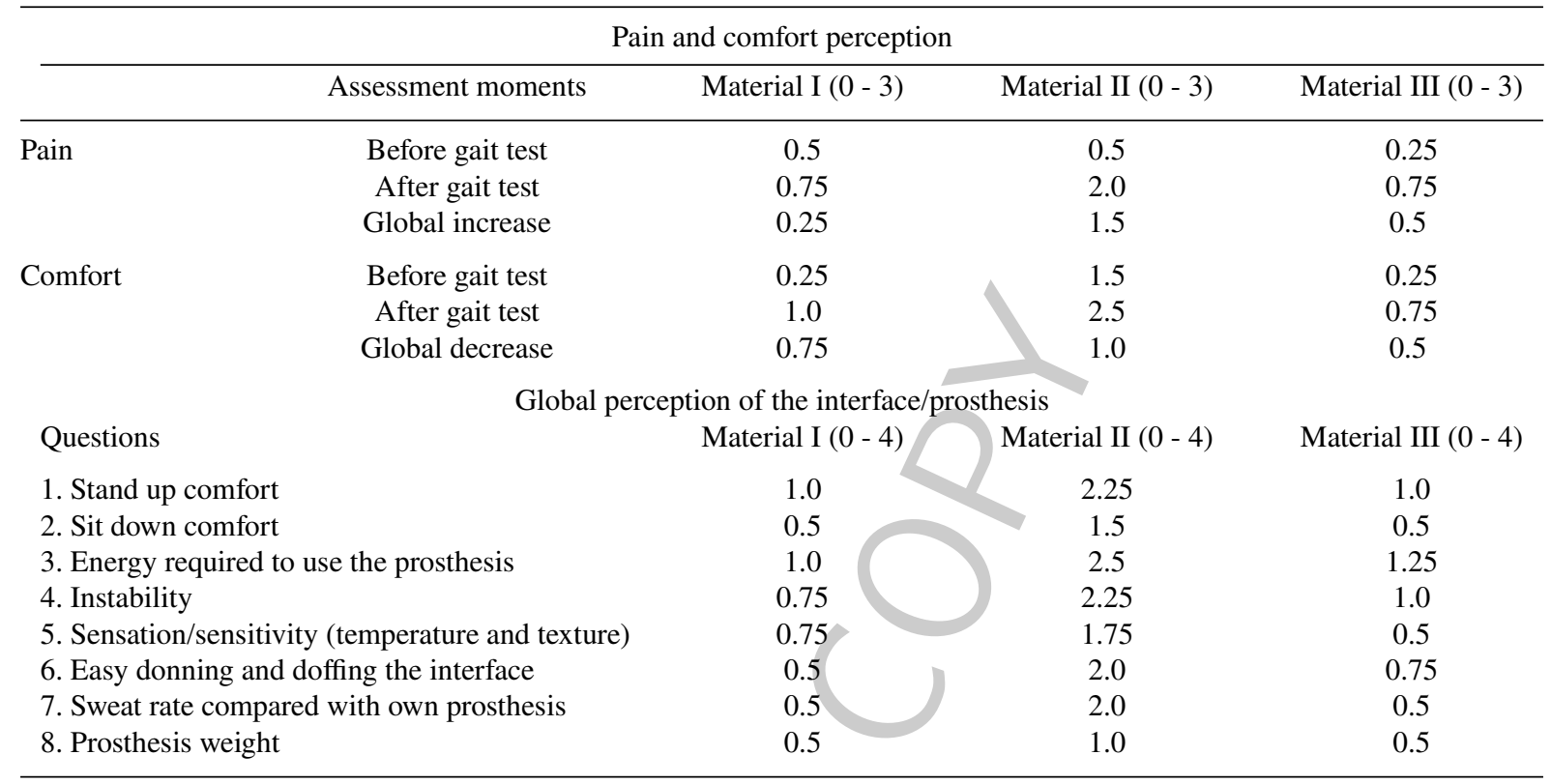

In the current study, all materials resulted in step length differences when averaged among all subjects and cadences. However, despite the lack of statistical significance, two different trends were observed. The use of Materials I and II resulted in a higher step length of the prosthetic leg than in the sound leg, with a higher difference for Material II. These results are in accordance with previously published results [20] in a gait study with transtibial amputees who used their own prosthesis, where slightly higher length differences were observed, probably because different protocols were used. Due to difficulties of the amputees in loading the weight on the prosthetic leg [5], the step length of the sound leg is normally lower. However, when Material III was used, results showed a higher step length for the sound leg than for the prosthetic leg. This shows that a single component of the prosthesis, in this case the interface, may allow an inversion of the symmetry effect in transtibial gait, and this is certainly the reason why the subjects perceived this material as the more comfortable (Table 6).

Asymmetry has also been described in gait temporal parameters for transtibial amputees comparatively with healthy subjects. In normal subjects, stance and swing phases are symmetric between legs and normally correspond to $60 \%$ of the gait cycle for the stance phase versus $40 \%$ for the swing phase $[2,20]$. In transtibial amputees, gait cycle asymmetries were found in the literature between stance and swing phases, when comparing the sound and prosthetic legs. For the prosthetic leg, the stance phase corresponded to $57-59 \%$ of the gait cycle and the swing phase was $43-41 \%$; in the sound leg, the stance phase was $63-$ $62 \%$ while the swing phase corresponded to $37-38 \%$ of the gait cycle $[2,20]$. The asymmetries between temporal gait phases, which had statistical significance in our study, could be caused by differences in step length and difficulty of weight loading in the stump, which lead to an increase of the stance phase of the sound leg and of the swing phase of the prosthetic leg.

Although the results of our study (Table 4) are globally in agreement with the above referred values [20] for all materials, Material III had the shortest double support time phase. 
Table 7

Summary of relevant properties of the interface materials previously assessed: bulk modulus $\left(E^{\prime}\right)$ tangent of the phase angle $(\tan \delta)$, thermal conductivity at $20^{\circ} \mathrm{C}(k)$, coefficient of friction (COF) and energy of adhesion measured on human skin (in vivo). Data collected from Refs $[15,16]$

\begin{tabular}{lccccc}
\hline & $\begin{array}{c}E^{\prime} \\
(\mathrm{MPa})\end{array}$ & $\tan \delta$ & $k(\mathrm{~W} /(\mathrm{m} . \mathrm{K}))$ & $\mathrm{COF}$ & $\begin{array}{c}\text { Energy of adhesion } \\
(\mathrm{mJ})\end{array}$ \\
\hline Material I & 1.62 & 0.27 & $0.1438 \pm 0.0002$ & $2.15 \pm 0.25$ & $0.133 \pm 0.007$ \\
Material II & 11.9 & 0.28 & $0.1996 \pm 0.0002$ & $1.91 \pm 0.09$ & $0.067 \pm 0.051$ \\
Material III & 1.64 & 0.57 & $0.1891 \pm 0.0003$ & $2.46 \pm 0.70$ & $0.340 \pm 0.322$ \\
\hline
\end{tabular}

GRF-V is largely studied and is believed to be particularly important for analysing shock absorption that occurs during the loading response phase of gait [20]. It is an important biomechanical parameter to analyse the amputee's risk of developing joint disorders in the residual limb and intact contralateral leg [10]. GRF-V increased with increasing cadence (Table 5) for all materials, as expected. In general, the GRF-V were asymmetric between legs, being higher in the sound leg, in agreement with previously published data [5,21].

The GRF-V in the prosthetic and sound legs registered in our study are within the range considered for gait in normal subjects, which is between $120 \%$ and $150 \%$ for walking cadences of $3.5 \mathrm{~km} / \mathrm{h}$ to $5 \mathrm{~km} / \mathrm{h}$ [20]. Further comparison between our GRF-V values and other previous published values is not possible, because other prosthesis components were used [21], or cadence and prosthetic components were not controlled [5]. Our GRF-V results globally differ between the studied materials. When just analysing the prosthetic limb, Material III damps more load at a slow cadence and Material II at normal and fast cadences. However, when analysing GRF-V of the prosthetic limb versus the sound limb, Material II is also responsible for the highest asymmetries of GRF-V between legs. The greater ability of Material II in damping load has a negative effect on the contralateral limb in both normal and fast cadences, being responsible for the highest absolute differences in GRF-V between limbs, which over time may have an adverse effect on the overloading of the sound leg [10]. For this material, there was a statistical significance between the slow and normal cadences and the slow and fast cadences, indicating the consistent sensibility of Material II.

Material III is responsible for the lowest GRF-V in the sound leg in all cadences, besides being the best damping at slow cadence and overall showed a more symmetric GRF-V distribution between legs. The interface material has cushioning and comfort purposes, reducing load transmission from the prosthesis to the residual limb [22]. Subjects preferred greater damping devices [23], which can explain subjects' better perception of Material III.

For the GRF-V gait parameter, although the results showed a clear direction of the differences, there was no significant difference between legs. This may be due to the small sample size.

The mechanical behavior of the three interface materials was analysed in a previous study [15] (Table 7), in which Material II showed to be the most rigid, with higher bulk modulus when compared to Materials I and III. Material III presented a higher $\tan \delta$ when compared to the other materials, corresponding to the highest hysteresis component on dynamic loading [15], and, therefore, to the highest energy dissipation/damping capacity. The more adequate mechanical behavior of Material III to accommodate soft tissues, bone prominences and dissipate loads, in combination with a higher coefficient of friction and adhesion, is in line with the current results of the perception of the amputees, since it was the material 
which provided the best sensation/sensitivity of temperature and texture and lower decrease of comfort (Table 6). Table 7 also shows that Material II presented a higher thermal conductivity value, as well as a higher stiffness and lower coefficient of friction and adhesion. This material was perceived by the subjects as the worst during its use (Table 6). Thus, it was concluded that the subjects' perception is mainly dependent on the mechanical properties of the interface.

\section{Conclusion}

In conclusion, it is considered that the prescription of Material I, due to its mechanical properties (similar stiffness and lower capacity of energy dissipation when compared to Material III), should be rethought to also include subjects with lower activity levels (K1-K2). On the other hand, Material II should only be prescribed for situations of non-daily use. The interfaces of Material III showed characteristics suitable for the activity levels for which it is recommended (K1-K2).

\section{Acknowledgements}

The authors thank IDEO SL (Seville, Spain) for providing the interface samples, the prosthesis components and the access to the subjects. The authors also acknowledge Instituto de Biomecánica y Postura de Sevilla (Seville, Spain) for allowing the gait study to be conducted in their laboratory. The authors furthermore dedicate special thanks to all subjects who contributed voluntarily to this study.

\section{Conflict of interest}

None to report.

\section{References}

[1] R. Sinha, W. Van Den Heuvel and P. Arokiasamy, Factors affecting quality of life in lower limb amputees, Prosthet Orthot Int 35 (2011), 90-96.

[2] J. Breakey, Gait of unilateral below-knee amputees, Orthotics and Prosthetics 30 (1976), 17-24.

[3] N. Vanicek, S. Strike, N. McNaughton and R. Polman, Gait patterns in transtibial amputee fallers vs. non-fallers: Biomechanical differences during level walking, Gait Posture 29 (2009), 415-420.

[4] A. Sadeeq, N. Osman, M. Naqshbandi, A. Eshaghi, M. Kamyab and H. Gholizadeh, Qualitative study of prosthetic suspension systems on transtibial amputees' satisfaction and perceived problems with their prosthetic devices, Arch Phys Med Rehabil 93 (2012), 1919-1923.

[5] I. Kovac, V. Medved and L. Ostojic, Ground reaction force analysis in traumatic transtibial amputees' gait, Coll Antropol 33 (2009), 107-114.

[6] B. Kolarova, M. Janura, Z. Svoboda and M. Elfmark, Limits of stability in persons with transtibial amputation with respect to prosthetic alignment alterations, Arch Phys Med Rehabil 94 (2013), 2234-2240.

[7] T. Lunsford and B. Contoyannis, Ciencia de los materiales, in: AAOS - Atlas de ortotesis y dispositivos de ayuda, J. Hsu, J. Michael and J. Fiks (eds), Elsevier, Barcelona, 2009, pp. 15-51.

[8] J. Berge, J. Czerniecki and G. Klute, Efficacy of shock-absorbing versus rigid pylons for impact reduction in transtibial amputees based on laboratory, field and outcome metrics, J Rehabil Res Dev 42 (2005), 795-808.

[9] G. Klute, G. Rowe, A. Mamishev and W. Ledoux, The thermal conductivity of prosthetic sockets and liners, Prosthet Orthot Int 31 (2007), 292-299. 
[10] D. Norvell, J. Czerniecki and G. Reiber, The prevalence of knee pain and symptomatic knee osteoarthritis among veteran traumatic amputees and nonamputees, Arch Phys Med Rehabil 86 (2005), 487-493.

[11] G. Klute, B. Glaister and J. Berge, Prosthetic liners for lower limb amputees: A review of the literature, Prosthet Orthot Int 34(2) (2010), 146-153.

[12] D. Selinger, Weight of Prefabricated Transtibial Gel Prosthetic Liners - A Standardized Sample - Resident Research Project, California State University, Dominguez Hills, California, 2004.

[13] H. Berke, Transtibial prostheses, in: Orthotics and Prosthetics in Rehabilitation, M. Lusardi and C. Nielsen (eds), Saunders Elsevier, Filadelfia, 2007, pp. 679-710.

[14] I. Kovac, K. Neven, Z. Ognjen, M. Vedrana, A. Mariana, V. Zoran, V. Tamara, I. Neven and L. Branko, Rehabilitation of lower limb amputees, Period. Biol. 117 (2015), 147-159.

[15] A. Cavaco, A. Ramalho, S. Pais and L. Durães, Mechanical and structural characterization of tibial prosthetic interfaces before and after aging under simulated service conditions, J Mech Behav Biomed Mater 43 (2015), 78-90.

[16] A. Cavaco, L. Durães, S. Pais and A. Ramalho, Friction of prosthetic interfaces used by transtibial amputees, Biotribology 6 (2016), 36-41.

[17] M. Castro, M. Figueiredo, S Abreu, H. Sousa, L. Machado, R. Santos and J. Vilas-Boas, The influence of gait cadence on the ground reaction forces and plantar pressures during load carriage of young adults, Appl Ergon 49 (2015), 41-46.

[18] D. Boone, T. Kobayashi, T. Chou, A. Arabian, K. Coleman, M. Orendurff and M. Zhang, Influence of malalignment on socket reaction moments during gait in amputees with transtibial prostheses, Gait Posture 37 (2013), 620-626.

[19] P. MacFarlane and M. Looney, Walkway length determination for steady state walking in young and older adults, Res $Q$ Exerc Sport 79 (2008), 261-267.

[20] I. Kovac, V. Medved and L. Ostojic, Spacial, temporal and kinematic characteristics of traumatic transtibial amputees' gait, Coll Antropol 34 (2010), 205-213.

[21] A. Eshraghi, N. Osman, M. Karimi, H. Gholizadeh, E. Soodmand and W. Abas, Gait biomechanics of individuals with transtibial amputation: Effect of suspension system, PLoS One 9 (2014), 1-12.

[22] E. Boutwell, R. Stine, A. Hansen, K. Tucker and S. Gard, Effect of prosthetic gel liner thickness on gait biomechanics and pressure distribution within the transtibial socket, J Rehabil Res Dev 49 (2012), 227-240.

[23] E. Viel and F. Plas, Atividades dos músculos durante o ciclo de marcha, in: A marcha humana, a corrida e o salto Biomecânica, investigações, normas e disfunções, E. Viel (ed.), Manole, Barueri, 2001, pp. 25-47. 